\title{
Automation of measurements permittivity of materials by the capacitor method
}

\author{
Andrey Zhukov ${ }^{1, *}$, Vasily Atamasov ${ }^{1}$, and Grigory Malenko ${ }^{1}$ \\ ${ }^{1}$ Tomsk State University, Faculty of Radiophysics, 36 Lenin Avenue, Tomsk 634050, Russian \\ Federation
}

\begin{abstract}
The article discusses an example of the automation of the process of measuring the dielectric constant of materials by the capacitor method using the LabVIEW graphical programming environment and the Agilent E4980A precision LCR meter. A description of the hardwaresoftware complex is presented, as well as the measurement results.
\end{abstract}

The modern development of science allows experimental studies of electromagnetic characteristics in frequency ranges previously unavailable for work. Especially important are such studies in the field of the electronic industry for the effective use of radio materials in the design of radio equipment and for predicting the behavior of radio components at certain frequencies [1]. Of interest are both promising and long-used materials. One of the most important electrophysical parameters is the dielectric constant of a substance. The equipment, which makes it possible to measure primary parameters for their further conversion into permittivity, is in great demand, since the determination of this value is required in many areas of human life.

Modern research laboratories annually replenish the fleet of measuring equipment to create automated measuring systems. Due to the correct selection of measuring tools and software used to collect, process and transmit data received from the measuring equipment, the whole process becomes more automated, which, in turn, increases the productivity of both the laboratory complex itself and the maintenance staff.

A high level of automation and system flexibility, as a rule, is achieved through software and universal measuring equipment. Communication between the computer and all other nodes and their compatibility is ensured by a combination of hardware, software and design tools.

One of such software products that allows you to: develop virtual measuring systems, ensure the interaction of measuring systems with a computer, enter and process measured values is the LabVIEW programming system $[2,3]$.

The authors proposed a way to use this platform as a software basis for automating the process of measuring dielectric constant using a precision LCR Agilent E4980A meter from the measuring equipment park of the collective usage center "TsKU of radio measurements of TSU".

* Corresponding author: gyk@mail.tsu.ru 
As standard, the Agilent E4980A precision LCR meter is capable of measuring various electrophysical parameters in the operating frequency range from $20 \mathrm{~Hz}$ to $2 \mathrm{MHz}$, followed by recording values on an external medium. The device is controlled by buttons located on its front panel.

The authors implemented in the LabVIEW system a program for automating the measurement process, averaging experimental values, converting averaged primary data to permittivities, and writing data to a text file.

The objects of research were foil dielectrics of the FLAN brand (foil sheet arylox filled) of a rectangular shape with a metal layer removed from the edges to minimize the influence of stray capacitances. All samples have similar geometric dimensions and differ only in dielectric material between the plates and the thickness of the dielectric layer. The layer thickness depends on the type of foiled dielectric, and the length and width of the metal plates of all samples are 5 and 4 centimeters, respectively.

For measurements, the capacitive method [5] should be chosen because of the ease of its practical implementation and the presence of a large amount of specialized literature. The real and imaginary parts of the impedance of the studied samples were measured in the range from $20 \mathrm{~Hz}$ to $2 \mathrm{MHz}$.

Recalculation of the primary data into the values of the parts of the dielectric constant was carried out by averaging over 16 measurements and further calculation using known formulas [4]:

$$
\begin{gathered}
\varepsilon^{\prime}=Z^{\prime \prime} /\left(\left(\left(Z^{\prime \prime}\right) 2+(Z) 2\right)\left(\omega \cdot C_{0}\right)\right) ; \\
\varepsilon^{\prime \prime}=Z /\left(\left(\left(Z^{\prime \prime}\right) 2+(Z) 2\right)\left(\omega \cdot C_{0}\right)\right) ; \\
C_{0}=\varepsilon_{0} \cdot S / d .
\end{gathered}
$$

Here $\varepsilon$ 'and $\varepsilon$ " are the real and imaginary parts of the dielectric constant, $Z$ and $Z$ " are the real and imaginary parts of the impedance, $C_{0}$ is the capacitance of the capacitor without a sample, $\omega$ is the cyclic frequency, $S$ is the area of the metal plates, $\mathrm{d}$ is the thickness of the dielectric layer .

An example of working with measuring equipment was taken on the official website of a manufacturer of measuring equipment. This virtual instrument allowed measurements to be made only at a fixed frequency. Also, it lacked the function of writing data to a file. The standard program was modified taking into account several measurements at the calibration frequencies of the device to further average the results and write the measured data to a file. A fragment of a block diagram is shown in Figure 1.

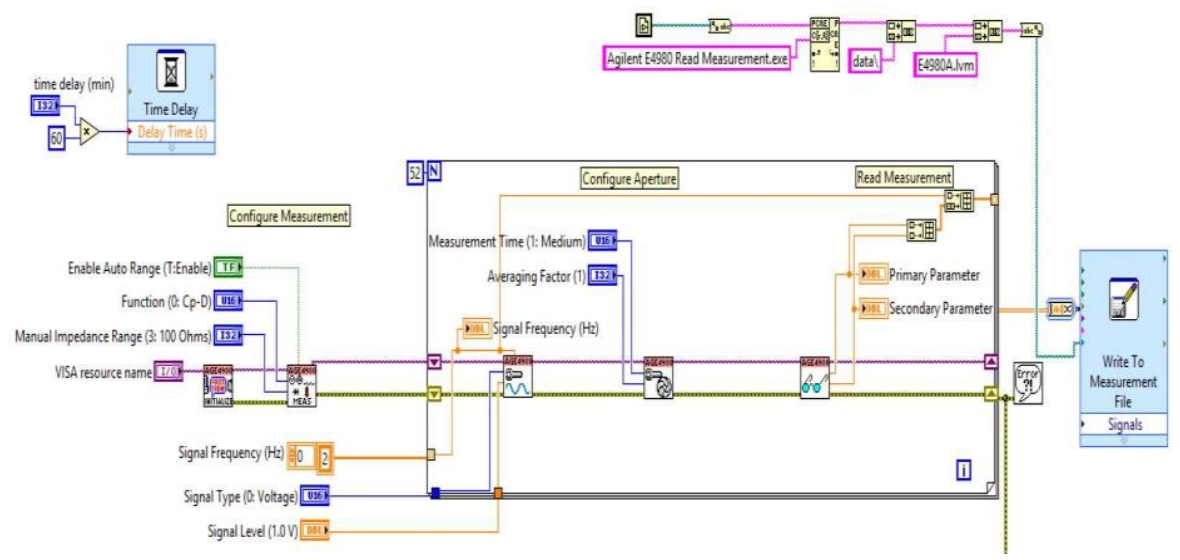

Fig. 1. Fragment block diagram of a program for measuring automation. 
As a result of the program on an external medium, a folder is created with the results of measurements of the real and imaginary parts of the impedance at the calibration frequencies of the precision meter. The number of text files in a folder depends on the selected number of measurements.

A separate program implements an algorithm for obtaining the average value of each measured parameter at given frequencies. Averaging was carried out over 16 measurements. The data obtained using formulas (1), (2) and (3) were converted into values of the real and imaginary parts of the dielectric constant and recorded in a separate text file.

Figure 2 shows the results of measuring the frequency dependence of the imaginary part of the dielectric constant of the investigated foil dielectrics, and table 1 presents the values of the real part of the dielectric constant of the substance on frequency.

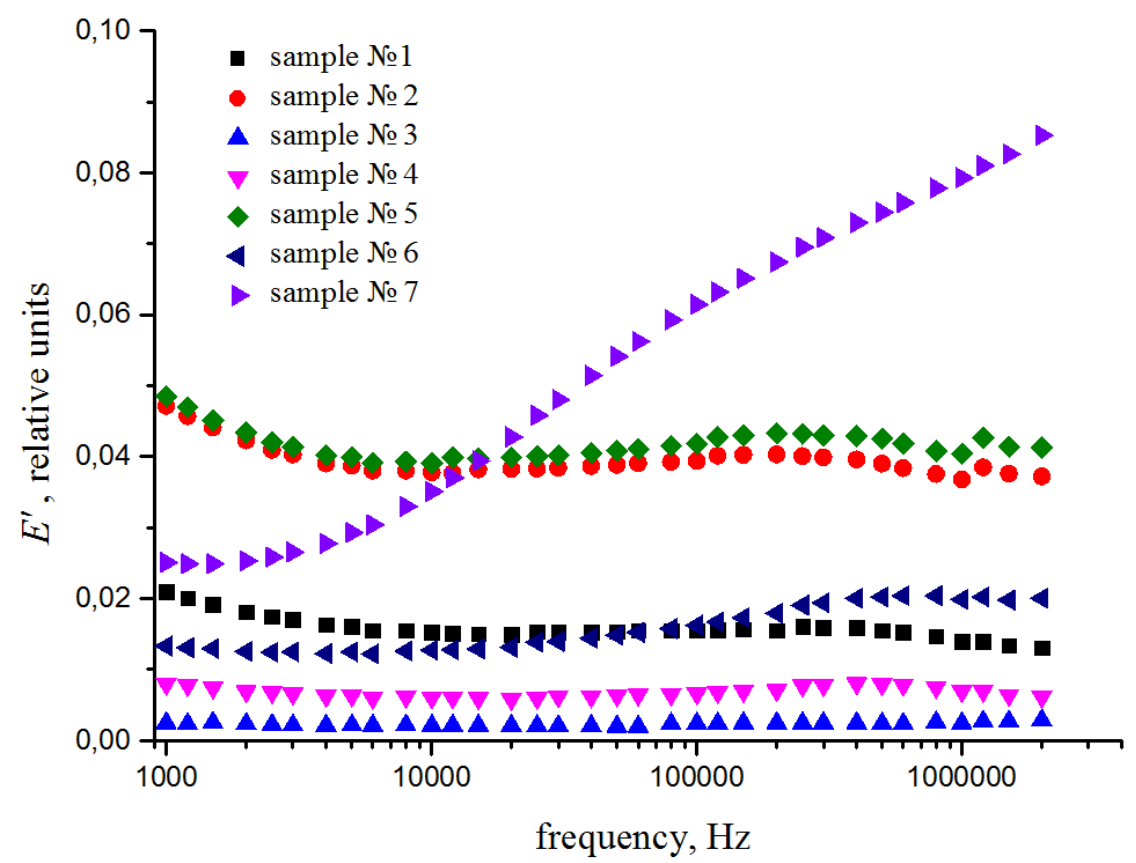

Fig. 2. Spectra of the imaginary part of the dielectric constant of the studied samples.

Table 1. Values of the real part of the dielectric constant of the studied samples.

\begin{tabular}{|l|c|c|c|c|c|c|c|}
\hline \\
Frequency
\end{tabular}


As a result of the work, the authors proposed a hardware-software complex for the automated measurement of permittivity spectra in the frequency range from $20 \mathrm{~Hz}$ to 2 $\mathrm{MHz}$ with subsequent recording of the measured values into a file.

The results of the work of the complex are presented on the example of the permittivity spectra of various foil dielectrics. This approach can significantly reduce the time for measurements and recalculation of data. Currently, work is underway to unify parts of the programs and unify the work of the complex.

\section{References}

1. A. Badyin, V. Vygovsky, A. Berdyugin, K. Dorozhkin , Russian Physics Journal, v. 60, pp. 62-64 (2017)

2. J. Travis, J. Kring, LabVIEW for everyone : graphical programming made easy and fun (NJ Prentice Hall, 2009)

3. J. Conway, St. Watts, A Software Engineering Approach to Labview (Prentice Hall, 2003)

4. V. A. Zhuravlev, V. I. Suslyaev, A. V. Zhuravlev and E. Yu. Korovin, Analysis of dielectric spectra of water with conductive impurities in a wide frequency range, Russian Physics Journal , v. 60, pp.1893-1900 (2018) 